\title{
Stability Analysis of an Improved HBV Model with CTL Immune Response
}

\author{
Mostafa Khabouze, ${ }^{1}$ Khalid Hattaf, ${ }^{1,2}$ and Noura Yousfi ${ }^{1}$ \\ ${ }^{1}$ Department of Mathematics and Computer Science, Faculty of Sciences Ben M'sik, Hassan II University, P.O. Box 7955, \\ Sidi Othman, Casablanca, Morocco \\ ${ }^{2}$ Centre Régional des Métiers de l'Education et de la Formation (CRMEF), Derb Ghalef, 20340 Casablanca, Morocco \\ Correspondence should be addressed to Khalid Hattaf; k.hattaf@yahoo.fr
}

Received 9 June 2014; Accepted 30 August 2014; Published 29 October 2014

Academic Editor: San-Lin You

Copyright (C) 2014 Mostafa Khabouze et al. This is an open access article distributed under the Creative Commons Attribution License, which permits unrestricted use, distribution, and reproduction in any medium, provided the original work is properly cited.

To better understand the dynamics of the hepatitis B virus (HBV) infection, we introduce an improved HBV model with standard incidence function, cytotoxic T lymphocytes (CTL) immune response, and take into account the effect of the export of precursor CTL cells from the thymus and the role of cytolytic and noncytolytic mechanisms. The local stability of the disease-free equilibrium and the chronic infection equilibrium is obtained via characteristic equations. Furthermore, the global stability of both equilibria is established by using two techniques, the direct Lyapunov method for the disease-free equilibrium and the geometrical approach for the chronic infection equilibrium.

\section{Introduction}

Currently, HBV infection is a major global health problem, which can lead to cirrhosis and liver cancer. From the World Health Organization (WHO), more than 240 million people have chronic (long-term) liver infections, and about 600, 000 people die every year due to the acute or chronic consequences of hepatitis B [1]. Therefore, many mathematical models have been developed in order to understand the dynamics of HBV infection. In this paper, we consider the model presented by Pang et al. in [2] that is given by the following nonlinear system of differential equations:

$$
\begin{aligned}
\dot{x} & =\lambda-d x-\beta v x+q y z, \\
\dot{y} & =\beta v x-a y-(p+q) y z, \\
\dot{v} & =k y-u v, \\
\dot{z} & =b+\frac{c y z}{\omega+y}-\mu z,
\end{aligned}
$$

where $x(t), y(t), v(t)$, and $z(t)$ are the numbers of uninfected target cells, infected cells, free virus, and CTL cells at time $t$, respectively. Susceptible host (healthy hepatocytes) cells are produced at a rate $\lambda$, die at a rate $d x$, and become infected by virus at a rate $\beta x v$. Infected cells die at a rate $a y$, return to the uninfected state by a nonlytic effector mechanism [3] at a rate $q y z$, and are killed by the CTL immune response at a rate $p y z$. Free virus is produced by infected cells at a rate $k y$ and decays at a rate $u v$. CTL cells expand in response to viral antigen derived from infected cells at a rate $c y z /(\omega+y)$, where $c$ is HBV-specific CTL stimulation rate and $\omega$ represents virus load for half-maximal CTL cells stimulation [4] and decay in the absence of antigenic stimulation at a rate $\mu z$. The parameter $b$ represents the export of precursor CTL cells from the thymus [4]. Note that the CTL immune response plays an important role in antiviral defense by killing infected cells and its effect has recently drawn much attention of many authors (see, e.g., [5-10]).

On the other hand, the authors Pang et al. [2] determined the basic reproduction number of system (1) as follows:

$$
R_{0}^{*}=\frac{\lambda}{d} \frac{\beta k}{u(a+(p+q)(b / \mu))} .
$$

As in $[10,11]$, we observe that $R_{0}^{*}$ is proportional to $\lambda / d$ which represents the number of total cells of the liver. This 
suggests that (1) may not be a reasonable model for describing $\mathrm{HBV}$ virus infection since it implies that an individual with a smaller liver may be more resistant to the virus infection than an individual with a larger one. Therefore, we propose the following model:

$$
\begin{aligned}
& \dot{x}=\lambda-d x-\frac{\beta v x}{x+y}+q y z, \\
& \dot{y}=\frac{\beta v x}{x+y}-a y-(p+q) y z, \\
& \dot{v}=k y-u v, \\
& \dot{z}=b+\frac{c y z}{\omega+y}-\mu z .
\end{aligned}
$$

In our case, the basic reproduction number is

$$
R_{0}=\frac{\beta k}{u(a+(p+q)(b / \mu))},
$$

which is independent of liver size.

The rest of this paper is organized as follows. In the next section, we show that our model is well posed by proving the existence, positivity, and boundedness of solutions of problem. Further, we determine the steady states of the model. In Section 3, we discuss the local stability of equilibria by analyzing the corresponding characteristic equations. The global stability of equilibria is analyzed in Section 4 . The paper ends with a conclusion and discussion in Section 5.

\section{Well Posedness and Steady States}

In this section, we will establish the positivity and boundedness of solutions of model (3), which imply that our model is well posed. Further, we will determine the steady states of the model.

2.1. Positivity and Boundedness of Solutions. First, we have the following result.

Theorem 1. All solutions starting from nonnegative initial conditions exist for all $t>0$ and remain bounded and nonnegative. Moreover, we have

(i) $T(t) \leq T(0)+\lambda / \delta$,

(ii) $v(t) \leq v_{0}+(k / u)\|y\|_{\infty}$,

(iii) $z(t) \leq z_{0}+b / \mu+(c / p \omega)\left(\lambda / \mu+x_{0}+y_{0}+\max (0,1-\right.$ $\left.d / \mu)\|x\|_{\infty}+\max (0,1-a / \mu)\|y\|_{\infty}\right)$,

where $T=x+y$ that represents the total cells of liver and $\delta=$ $\min (a, d)$

Proof. For the positivity, we show that any solution starting in nonnegative orthant $\mathbb{R}_{+}^{4}=\left\{(x, y, v, z) \in \mathbb{R}^{4}: x \geq 0\right.$, $y \geq 0, v \geq 0, z \geq 0\}$ remains there forever. In fact, $(x(t), y(t), v(t), z(t)) \in \mathbb{R}_{+}^{4}$ and we have

$$
\begin{aligned}
& \left.\dot{x}\right|_{x=0}=\lambda+q y z \geq 0, \\
& \left.\dot{y}\right|_{y=0}=\beta v \geq 0, \\
& \left.\dot{v}\right|_{v=0}=k y \geq 0, \\
& \left.\dot{z}\right|_{z=0}=b>0 .
\end{aligned}
$$

Hence, the positivity of all solutions initiating in $\mathbb{R}_{+}^{4}$ is guaranteed.

Now, we prove that the solutions are bounded.

As $\dot{T} \leq \lambda-d x-a y$, we deduce that

$$
T(t) \leq T(0) e^{-\delta t}+\frac{\lambda}{\delta}\left(1-e^{-\delta t}\right),
$$

since $0 \leq e^{-\delta t} \leq 1$ and $1-e^{-\delta t} \leq 1$, we get (i).

Next, we show (ii). The equation, $\dot{v}=k y-u v$, implies that

$$
v(t)=v_{0} e^{-u t}+k \int_{0}^{t} y(s) e^{(s-t) u} d s .
$$

Then,

$$
v(t) \leq v_{0}+\frac{k}{u}\|y\|_{\infty}\left(1-e^{-t u}\right) .
$$

Since $1-e^{-t \mu} \leq 1$, we deduce (ii).

Finally, we show (iii). From the fourth equation of (3), we get

$$
\dot{z}+\mu z \leq b+\frac{c}{\omega} y z
$$

Hence,

$$
\dot{z}+b z \leq b+\frac{c}{p \omega}[\lambda-(\dot{x}+d x)-(\dot{y}+a y)] .
$$

Thus,

$$
\begin{aligned}
z(t) e^{\mu t}-z_{0} \leq & \left(\frac{b}{\mu}+\frac{c \lambda}{p \omega \mu}\right)\left(e^{\mu t}-1\right) \\
& -\frac{c}{p \omega}\left[\int_{0}^{t} e^{(\mu-d) s} \frac{d}{d s}\left(x(s) e^{d s}\right) d s\right. \\
& \left.+\int_{0}^{t} e^{(\mu-a) s} \frac{d}{d s}\left(y(s) e^{a s}\right) d s\right] .
\end{aligned}
$$

Using the integration by parts, we get

$$
\begin{aligned}
& \int_{0}^{t} e^{(\mu-d) s} \frac{d}{d s}\left(x(s) e^{d s}\right) d s \\
& \quad=\left[x(s) e^{\mu s}\right]_{0}^{t}-(\mu-d) \int_{0}^{t} x(s) e^{\mu s} d s, \\
& \int_{0}^{t} e^{(\mu-a) s} \frac{d}{d s}\left(y(s) e^{a s}\right) d s \\
& \quad=\left[x(s) e^{\mu s}\right]_{0}^{t}-(\mu-a) \int_{0}^{t} y(s) e^{\mu s} d s .
\end{aligned}
$$


Hence,

$$
\begin{gathered}
z(t) \leq\left[\frac{c}{p \omega}\left(x_{0}+y_{0}\right)+z_{0}\right] e^{-\mu t}+\left(\frac{b}{\mu}+\frac{c \lambda}{p \omega \mu}\right)\left(1-e^{-\mu t}\right) \\
+\frac{c}{p \omega}\left\{\int_{0}^{t}[(\mu-d) x(s)+(\mu-a) y(s)] e^{\mu(s-t)} d s\right. \\
-x(t)-y(t)\} .
\end{gathered}
$$

If $\mu-d \leq 0$ and $\mu-a \leq 0$, we have

$$
z(t) \leq z_{0}+\frac{b}{\mu}+\frac{c}{p \omega}\left(\frac{\lambda}{\mu}+x_{0}+y_{0}\right) .
$$

If $\mu-d \leq 0$ and $\mu-a \geq 0$, we have

$$
z(t) \leq z_{0}+\frac{b}{\mu}+\frac{c}{p \omega}\left[\frac{\lambda}{\mu}+x_{0}+y_{0}+\left(1-\frac{a}{\mu}\right)\|y\|_{\infty}\right] .
$$

If $\mu-d \geq 0$ and $\mu-a \leq 0$, we have

$$
z(t) \leq z_{0}+\frac{b}{\mu}+\frac{c}{p \omega}\left[\frac{\lambda}{\mu}+x_{0}+y_{0}+\left(1-\frac{d}{\mu}\right)\|x\|_{\infty}\right] .
$$

If $\mu-d \geq 0$ and $\mu-a \geq 0$, we have

$$
\begin{gathered}
z(t) \leq z_{0}+\frac{b}{\mu}+\frac{c}{p \omega}\left[\frac{\lambda}{\mu}+x_{0}+y_{0}+\left(1-\frac{d}{\mu}\right)\|x\|_{\infty}\right. \\
\left.+\left(1-\frac{a}{\mu}\right)\|y\|_{\infty}\right] .
\end{gathered}
$$

From (14)-(17), we deduce (iii).

2.2. Steady States. In this subsection, we show that there exist a disease-free equilibrium and one infection equilibrium which represents the chronic infection equilibrium.

It is not hard to see that if $R_{0} \leq 1$, the disease-free steady state $E_{f}(\lambda / d, 0,0, b / \mu)$ is the unique steady state, corresponding to the extinction of the free virus. The following result presents the existence and uniqueness of endemic equilibrium when $R_{0}>1$.

\section{Theorem 2.}

(1) If $R_{0} \leq 1$, then the system (3) has a unique disease-free equilibrium of the form $E_{f}(\lambda / d, 0,0, b / \mu)$.

(2) If $R_{0}>1$, then the system (3) has a unique chronic infection equilibrium of the form $E^{*}\left(x^{*}, y^{*}, v^{*}, z^{*}\right)$ with $x^{*}>0, y^{*}>0, v^{*}>0$, and $z^{*}>0$.

Proof. At any equilibrium, the following equations hold:

$$
\begin{gathered}
\lambda-d x-\frac{\beta v x}{x+y}+q y z=0, \\
\frac{\beta v x}{x+y}-a y-(p+q) y z=0, \\
k y-u v=0 \\
b+\frac{c y z}{\omega+y}-\mu z=0 .
\end{gathered}
$$

By (18), we get

$$
\begin{aligned}
& v=\frac{k y}{u}, \\
& z=\frac{b(\omega+y)}{\mu \omega+(\mu-c) y}, \\
& x=f(y),
\end{aligned}
$$

where

$$
f(y)=\frac{\lambda}{d}-y\left(\frac{a}{d}+\frac{p b(\omega+y)}{d \mu \omega+d(\mu-c) y}\right) .
$$

Hence, we obtain the following equation:

$$
\frac{\beta k}{u} \frac{f(y)}{f(y)+y}=a+\frac{b(p+q)(\omega+y)}{\mu \omega+(\mu-c) y} .
$$

Now, we consider the function $g$ defined on $[0,+\infty[-\{\mu \omega /(c-\mu)\}$ by

$$
g(y)=\frac{\beta k}{u} \frac{f(y)}{f(y)+y}-a-\frac{b(p+q)(\omega+y)}{\mu \omega+(\mu-c) y} .
$$

We have $g(0)=(\beta k / u)\left(1-1 / R_{0}\right)>0$ and

$$
\begin{gathered}
f^{\prime}(y)=-\left(\frac{a}{d}+\frac{p b(\omega+y)}{d \mu \omega+d(\mu-c) y}\right) \\
-\frac{d c p b \omega y}{[d \mu \omega+d(\mu-c) y]^{2}}<0, \\
g^{\prime}(y)=\frac{\beta k\left(y f^{\prime}(y)-f(y)\right)}{u(f(y)+y)^{2}}-\frac{(p+q) c b \omega y}{[\mu \omega+(\mu-c) y]^{2}}<0 .
\end{gathered}
$$

Let $\alpha=\mu \omega /(c-\mu)$ be a pole of $g$; then, we discuss two cases.

(i) If $c>\mu$, then $\alpha>0$. As $z=b(\omega+y) /(\mu \omega+(\mu-c) y) \geq 0$ we deduce that $y<\alpha$. Hence there is no equilibrium point if $y \geq \alpha$. It is easy to show that

$$
\lim _{y \rightarrow \alpha^{-}} g(y)=-\infty
$$

Then the function $g$ admits a unique root $y^{*}$ on interval $] 0, \alpha$, since $f(0)=\lambda / d>0$ and $\lim _{y \rightarrow \alpha^{-}} f(y)=$ $-\infty$. So, there exists a unique $\tilde{y} \in] 0, \alpha[$ such that $f(\widetilde{y})=0$. We have

$$
g(\tilde{y})=-a-\frac{b(p+q)(\omega+\tilde{y})}{\mu \omega+(\mu-c) \tilde{y}}<0 .
$$

We deduce that $0<y^{*}<\tilde{y}$; this implies that $f(\tilde{y})<$ $f\left(y^{*}\right)<f(0)$ because $f$ is decreasing on $] 0, \alpha[$. Then $0<x^{*}<\lambda / d$. Clearly $v^{*}$ and $z^{*}$ are positive.

Hence, there exists a unique endemic $E^{*}\left(x^{*}, y^{*}, v^{*}, z^{*}\right)$ with $\left.y^{*} \in\right] 0, \tilde{y}\left[, x^{*}>0, v^{*}>0\right.$, and $z^{*}>0$. 
(ii) If $c<\mu$, then $\alpha<0$ and $\lim _{y \rightarrow+\infty} f(y)=-\infty$. Since $f(0)=\lambda / d>0$, hence there exists a unique $\bar{y} \in] 0,+\infty[$ such that $f(\bar{y})=0$.

We have

$$
g(\bar{y})=-a-\frac{b(p+q)(\omega+\bar{y})}{\mu \omega+(\mu-c) \bar{y}}<0 .
$$

Using the same technique, we deduce that $x^{*}, v^{*}$, and $z^{*}$ are positive.
Thus, there exists a unique endemic $E^{*}\left(x^{*}, y^{*}, v^{*}, z^{*}\right)$ with $\left.y^{*} \in\right] 0, \bar{y}\left[, x^{*}>0, v^{*}>0\right.$, and $z^{*}>0$.

This proves the theorem.

\section{Local Stability of Equilibria}

Let $E(x, y, v, z)$ be any arbitrary equilibrium. Then the characteristic equation about $E$ is given by

$$
\left|\begin{array}{cccc}
-d-\frac{\beta v y}{(x+y)^{2}}-\xi & \frac{\beta x v}{(x+y)^{2}}+q z & -\frac{\beta x}{x+y} & q y \\
\frac{\beta v y}{(x+y)^{2}} & -\frac{\beta x v}{(x+y)^{2}}-a-(p+q) z-\xi & \frac{\beta x}{x+y} & -(p+q) y \\
0 & k & -u-\xi & 0 \\
0 & \frac{c \omega z}{(\omega+y)^{2}} & 0 & \frac{c y}{\omega+y}-\mu-\xi
\end{array}\right|=0 .
$$

The characterization of the local stability of the disease-free equilibrium is given by the following statement.

Theorem 3. Let us define $R_{0}=\beta k /(u(a+(p+q)(b / \mu)))$.

(i) If $R_{0}<1$, then $E_{f}$ is locally asymptotically stable.

(ii) If $R_{0}>1$, then $E_{f}$ is unstable.

Proof. At $E_{f}$, (27) reduces to

$$
(\mu+\xi)(\xi+d)\left[\xi^{2}+\left(u+\frac{\beta k}{u R_{0}}\right) \xi+\frac{\beta k}{R_{0}}\left(1-R_{0}\right)\right]=0,
$$

where the roots are

$$
\begin{aligned}
\xi_{1}= & -\mu, \\
\xi_{2}= & -d, \\
\xi_{3}= & -\left(u+\frac{\beta k}{u R_{0}}\right) \\
& \left.-\sqrt{\left(u+\frac{\beta k}{u R_{0}}\right)^{2}-4 \frac{\beta k}{R_{0}}\left(1-R_{0}\right)}\right)(2)^{-1}, \\
\xi_{4}= & -\left(u+\frac{\beta k}{u R_{0}}\right) \\
& \left.+\sqrt{\left(u+\frac{\beta k}{u R_{0}}\right)^{2}-4 \frac{\beta k}{R_{0}}\left(1-R_{0}\right)}\right)(2)^{-1} .
\end{aligned}
$$

It is clear that $\xi_{1}, \xi_{2}$, and $\xi_{3}$ are negative. Moreover, $\xi_{4}$ is negative when $R_{0}<1$; thus, $E_{f}$ is locally asymptotically stable.
Now, we focus on local stability of the chronic infection equilibrium $E^{*}$. It is easy to verify that the point $E^{*}$ does not exist if $R_{0}<1$ and $E^{*}=E_{f}$ when $R_{0}=1$. If $R_{0}>1$, then we have the following theorem.

Theorem 4. If $R_{0}>1$, then the chronic infection equilibrium $E^{*}$ is locally asymptotically stable.

Proof. We assume that $R_{0}>1$. At $E^{*},(27)$ reduces to

$$
\xi^{4}+a_{1} \xi^{3}+a_{2} \xi^{2}+a_{3} \xi+a_{4}=0
$$

where

$$
\begin{aligned}
& a_{1}=u+N+\phi_{1}, \\
& a_{2}=N\left(u+\phi_{1}\right)+u\left(d+\frac{\beta v^{*}}{x^{*}+y^{*}}\right)+\phi_{2}+M y^{*}(p+q), \\
& a_{3}=N\left(u \phi_{1}+\phi_{2}\right)+u \phi_{3}+\phi_{4}+M y^{*} u(p+q), \\
& a_{4}=u\left(N \phi_{3}+\phi_{4}\right),
\end{aligned}
$$

with

$$
\begin{aligned}
& M=\frac{c \omega z^{*}}{\left(\omega+y^{*}\right)^{2}}, \\
& N=\mu-\frac{c y^{*}}{\omega+y^{*}},
\end{aligned}
$$




$$
\begin{aligned}
& C_{1}=\frac{\beta v^{*} y^{*}}{\left(x^{*}+y^{*}\right)^{2}}, \\
& C_{2}=\frac{\beta v^{*} x^{*}}{\left(x^{*}+y^{*}\right)^{2}}+q z^{*}, \\
& \phi_{1}=a+d+C_{1}+C_{2}+p z^{*}, \\
& \phi_{2}=d C_{2}+\left(d+C_{1}\right)\left(a+p z^{*}\right), \\
& \phi_{3}=C_{1}\left(a+p z^{*}\right)+\frac{d \beta v^{*}}{x^{*}+y^{*}}, \\
& \phi_{4}=M y^{*}\left[(p+q) d+p C_{1}\right] .
\end{aligned}
$$

Clearly when $R_{0}>1, a_{1}, a_{2}, a_{3}$, and $a_{4}$ are positive. In addition,

$$
\begin{aligned}
\left|\begin{array}{cc}
a_{1} & 1 \\
a_{3} & a_{2}
\end{array}\right|= & u d\left(C_{2}+a+d+p z^{*}\right) \\
& +N\left(a u+a d+a_{2}+\phi_{2}+d \phi_{1}\right) \\
& +d \phi_{2}+a_{2}\left(a+C_{2}\right)+C_{1}\left(a_{2}-p M y^{*}\right)>0 .
\end{aligned}
$$

In the same manner, we have

$$
\left|\begin{array}{ccc}
a_{1} & 1 & 0 \\
a_{3} & a_{2} & a_{1} \\
0 & a_{4} & a_{3}
\end{array}\right|=a_{3}\left|\begin{array}{cc}
a_{1} & 1 \\
a_{3} & a_{2}
\end{array}\right|-a_{1}^{2} a_{4}>0 .
$$

From the Routh-Hurwitz theorem given in [12], all roots of (30) have negative real parts. Then $E^{*}$ is locally asymptotically stable when $R_{0}>1$.

\section{Global Stability of Equilibria}

In this section, we establish the global stability of the equilibria. Firstly, we have the following result.

Theorem 5. The disease-free equilibrium $E_{f}$ is globally asymptotically stable when $R_{0} \leq 1$.

Proof. Define

$$
D=\left\{(x, y, v, z) \in \mathbb{R}_{+}^{4}: z \geq \frac{b}{\mu}\right\} .
$$

We see that any solution $(x(t), y(t), v(t), z(t))$ starting in $D$ remains there forever. Indeed, from Theorem 1 we get that $(x(t), y(t), v(t), z(t)) \in \mathbb{R}_{+}^{4}$. It remains to prove that $z(t) \geq b / \mu$ with $z_{0} \geq b / \mu$. From the fourth equation of (3), we get

$$
z(t) \geq \frac{b}{\mu}+\left(z_{0}-\frac{b}{\mu}\right) e^{-\mu t} .
$$

This implies that $z(t) \geq b / \mu$. Hence $(x(t), y(t), v(t), z(t)) \in D$.
If $R_{0} \leq 1$, let us define a function $V$ on $D$ as follows:

$$
V=y+\frac{\beta}{u R_{0}} v
$$

Calculating the time derivative of $V$ along the solution of (3), we obtain

$$
\begin{aligned}
\dot{V} & =\frac{\beta}{R_{0}}\left(\frac{x}{x+y} R_{0}-1\right) v-(p+q)\left(z-\frac{b}{\mu}\right) y, \\
& \leq \frac{\beta}{R_{0}}\left(R_{0}-1\right) v .
\end{aligned}
$$

Since $R_{0} \leq 1$, then $\dot{V} \leq 0$. Furthermore, if $S$ is the set of solutions of the system, where $\dot{V}=0$, then the LyapunovLaSalle theorem [13] implies that all paths in $D$ approach the largest positively invariant subset of the set $S$. Here, $S$ is the set, where $v=0$. On the boundary of $D$, where $v=0$, we have $y=0, \dot{x}=\lambda-d x$, and $\dot{z}=b-\mu z$. Then

$$
\lim _{t \rightarrow+\infty} x(t)=\frac{\lambda}{d}, \quad \lim _{t \rightarrow+\infty} z(t)=\frac{b}{\mu} .
$$

Thus, all solution paths in $D$ approach the disease-free equilibrium $E_{f}$ when $R_{0} \leq 1$. Hence, $E_{f}$ is globally asymptotically stable in $D$.

To study the global stability of the chronic infection equilibrium, we will use the geometric approach defined by Li and Muldowney in [14]. A short overview of this geometric approach can be found in [15-17]. In a more simple way, Theorem 5.3 in [14] requires three conditions ensuring that global stability of a given equilibrium point is verified.

The first condition is the existence of a unique locally stable endemic equilibrium. Indeed, as proved in Theorem 4 from this paper, $E^{*}$ is the unique locally stable endemic equilibrium when $R_{0}>1$.

The second condition is the existence of a compact set in the interior of the definition domain of the solutions $D$ defined in the proof of Theorem 5, which is absorbing for the system (3). This is equivalent as shown in [18] to the uniform persistence of the state variables and the boundness of $D$. In our case, we proved in Theorem 1 that all solutions in system (3) are bounded. Thus the set $D$ is also bounded. Further, we have proved in Theorem 3 that the disease-free equilibrium $E_{f}$ is unstable if $R_{0}>1$. This instability of $E_{f}$ on $\partial D$ implies the uniform persistence [19]. 
The third condition is the fulfillment of the Bendixson criterion [14]. In order to verify this third condition, we consider the following subsystem of (3):

$$
\begin{aligned}
& \dot{x}=\lambda-d x-\frac{\beta v x}{x+y}+q y z, \\
& \dot{y}=\frac{\beta v x}{x+y}-a y-(p+q) y z, \\
& \dot{v}=k y-u v .
\end{aligned}
$$

$$
J^{[2]}=\left(\begin{array}{ccc}
-d-\frac{\beta v}{x+y}-a-(p+q) z & \frac{\beta x}{x+y} & \frac{\beta x}{x+y} \\
k & -d-\frac{\beta v y}{(x+y)^{2}}-u & \frac{\beta v x}{(x+y)^{2}}+q z \\
0 & \frac{\beta v y}{(x+y)^{2}} & -\frac{\beta v x}{(x+y)^{2}}-a-(p+q) z-u
\end{array}\right) .
$$

In this case, we choose $P=\operatorname{diag}(1, y / v, y / v)$. Hence,

$$
P_{f} P^{-1}=\operatorname{diag}\left(0, \frac{\dot{y}}{y}-\frac{\dot{v}}{v}, \frac{\dot{y}}{y}-\frac{\dot{v}}{v}\right),
$$

$$
\begin{aligned}
& B_{11}=-d-\frac{\beta v}{x+y}-a-(p+q) z, \\
& B_{12}=\left(\frac{\beta v x}{y(x+y)} \frac{\beta v x}{y(x+y)}\right), \\
& B_{21}=\left(\begin{array}{c}
k \frac{y}{v} \\
0
\end{array}\right), \\
& B_{22}=\left(\begin{array}{c}
\frac{\dot{y}}{y}-\frac{\dot{v}}{v}-d-u-\frac{\beta v y}{(x+y)^{2}} \\
\frac{\beta v y}{(x+y)^{2}}
\end{array}\right.
\end{aligned}
$$

Let $\left(w_{1}, w_{2}, w_{3}\right)$ be a vector in $\mathbb{R}^{3}$; choose a norm in $\mathbb{R}^{3}$ defined as follows: $\left|w_{1}, w_{2}, w_{3}\right|=\max \left\{\left|w_{1}\right|,\left|w_{2}\right|+\left|w_{3}\right|\right\}$ and let $\mu$ be the Lozinskii measure with respect to this norm. Then we have the following estimate; see [20]:

$$
\mu(B) \leq \sup \left\{g_{1}, g_{2}\right\}
$$

where $g_{1}=\mu_{1}\left(B_{11}\right)+\left|B_{12}\right|$ and $g_{2}=\left|B_{21}\right|+\mu_{1}\left(B_{22}\right)$; here $\mu_{1}$ denotes the Lozinskii measure with respect to $l_{1}$ vector norm
The Jacobian matrix of system (40) is

$$
J=\left(\begin{array}{ccc}
-d-\frac{\beta v y}{(x+y)^{2}} & \frac{\beta v x}{(x+y)^{2}}+q z & -\frac{\beta x}{x+y} \\
\frac{\beta v y}{(x+y)^{2}} & -\frac{\beta v x}{(x+y)^{2}}-a-(p+q) z & \frac{\beta x}{x+y} \\
0 & k & -u
\end{array}\right)
$$

and its second addictive compound matrix is where matrix $P_{f}$ is obtained by replacing each entry $p_{i j}$ of $P$ by its derivative in the direction of solution of (40). Moreover, we have

$$
B=P_{f} P^{-1}+P J^{[2]} P^{-1}=\left(\begin{array}{ll}
B_{11} & B_{12} \\
B_{21} & B_{22}
\end{array}\right),
$$

where and $\left|B_{12}\right|$ and $\left|B_{21}\right|$ are matrix norms with respect to $l_{1}$ norm. Moreover, we have

$$
\begin{aligned}
\mu_{1}\left(B_{11}\right) & =-d-\frac{\beta v}{x+y}-a-(p+q) z, \\
\left|B_{12}\right| & =\frac{\beta v x}{y(x+y)}=\frac{\dot{y}}{y}+a+(p+q) z, \\
\left|B_{21}\right| & =\frac{k y}{v}=\frac{\dot{v}}{v}+u,
\end{aligned}
$$




$$
\begin{aligned}
\mu_{1}\left(B_{22}\right) & =\max \left\{\frac{\dot{y}}{y}-\frac{\dot{v}}{v}-d-u, \frac{\dot{y}}{y}-\frac{\dot{v}}{v}-a-u-(p+q) z\right\} \\
& \leq \frac{\dot{y}}{y}-\frac{\dot{v}}{v}-u-\delta .
\end{aligned}
$$

Hence, we obtain

$$
\begin{aligned}
& g_{1}=\frac{\dot{y}}{y}-d-\frac{\beta v}{x+y}, \\
& g_{2} \leq \frac{\dot{y}}{y}-\delta .
\end{aligned}
$$

Therefore,

$$
\mu(B) \leq \frac{\dot{y}}{y}-\delta
$$

Consequently,

$$
\bar{q}_{2}=\lim _{t \rightarrow \infty} \sup \sup _{X_{0} \in K} \frac{1}{t} \int_{0}^{t} \mu(B) d s \leq-\frac{\delta}{2}<0,
$$

which implies that the third condition is realized. Hence, the conditions of Theorem 3.5 in [14] are fulfilled; consequently, the endemic equilibrium $\left(x^{*}, y^{*}, v^{*}\right)$ of the subsystem $(40)$ is globally asymptotically stable.

Now, consider the fourth equation of system (3)

$$
\dot{z}=b+\frac{c y z}{\omega+y}-\mu z,
$$

and its limit system is

$$
\dot{z}=b+\frac{c y^{*} z}{\omega+y^{*}}-\mu z .
$$

Since $\mu-c y^{*} /\left(\omega+y^{*}\right)=b / z^{*}$, we get

$$
\dot{z}=b\left(1-\frac{z}{z^{*}}\right) \text {. }
$$

Therefore,

$$
\lim _{t \rightarrow \infty} z(t)=z^{*}
$$

Thus, the endemic equilibrium $E^{*}$ is globally asymptotically stable.

Summarizing the above, we have established the following result.

Theorem 6. The chronic infection equilibrium $E^{*}$ is globally asymptotically stable if $R_{0}>1$.

\section{Conclusion and Discussion}

In this paper, we have presented a mathematical model based on a nonlinear system of differential equations. The population cells were partitioned into four classes, uninfected target cells, infected cells, free virus, and CTL cells. The basic reproduction number $R_{0}$ corresponding to our model is independent of the liver size. Then, our model is more reasonable than the model presented in [2] to describe the $\mathrm{HBV}$ infection. In addition, we have proved the existence, positivity, and the boundedness of solutions of the problem, which implies that the model is well posed. By analyzing the model, we have shown that the disease-free equilibrium $E_{f}$ is globally asymptotically stable if the basic reproduction number satisfies $R_{0} \leq 1$, which leads to the eradication of virus from the liver. When $R_{0}>1$, the disease-free equilibrium becomes unstable and a unique chronic infection equilibrium exists and is globally asymptotically stable. In this case, the virus persists in the population.

From our main results summarized above, we conclude that the dynamical behavior of our model is completely determined by the basic reproduction number $R_{0}$. This allows determining the strategies to control the HBV infection by reducing the value of $R_{0}$ to below or equal one (the case when $E_{f}$ is globally asymptotically stable). From the explicit formula (4) for $R_{0}$, we see that $R_{0}$ can be decreased by increasing the export of precursor CTL cells from the thymus and both cytolytic and noncytolytic mechanisms. This observation shows that the CTL immune response plays a critical role in eradication of virus from the liver. On the other hand, $R_{0}$ can be decreased by decreasing the parameters $\beta$ and $k$ which represent the rates of infection and production of virus, respectively. To do this biologically, we improve better our model by introducing the nucleoside analogues lamivudine or adefovir dipivoxil drug treatment in order to stop the virus from replicating. In addition, nucleoside analogues may also interfere with de novo infection of hepatocytes by hindering the transformation of relaxed circular DNA into cccDNA [21]. So, under therapy both production rate of new virions $(k)$ and the rate of de novo infection $(\beta)$ are reduced. Consequently, our model becomes

$$
\begin{aligned}
& \dot{x}=\lambda-d x-(1-\eta) \frac{\beta v x}{x+y}+q y z, \\
& \dot{y}=(1-\eta) \frac{\beta v x}{x+y}-a y-(p+q) y z, \\
& \dot{v}=(1-\epsilon) k y-u v, \\
& \dot{z}=b+\frac{c y z}{\omega+y}-\mu z,
\end{aligned}
$$

where the parameters $\eta$ and $\epsilon$ measure the efficacy of the therapy. An efficacy of $0(0 \%)$ denotes that there is no inhibition, whereas an efficacy of 1 (100\%) denotes complete inhibition. The basic reproduction number $R_{0 T}$ under therapy becomes

$$
R_{0 T}=\frac{(1-\epsilon)(1-\eta) \beta k}{u(a+(p+q)(b / \mu))}
$$

which implies that the basic reproduction number can be decreased by increasing the efficacy of drug treatment. Therefore, the results obtained from this work can be useful to determine an effective treatment against the hepatitis B virus. 


\section{Conflict of Interests}

The authors declare that they have no conflict of interests regarding the publication of this paper.

\section{Acknowledgment}

The authors thank the editor and the anonymous referees for very helpful suggestions and comments which have improved the presentation and content of the paper.

\section{References}

[1] WHO, "Hepatitis B," Fact sheet 204, 2013, http://www.who.int/ mediacentre/factsheets/fs204/en/.

[2] J. Pang, J.-A. Cui, and J. Hui, "The importance of immune responses in a model of hepatitis B virus," Nonlinear Dynamics, vol. 67, no. 1, pp. 723-734, 2012.

[3] C. Long, H. Qi, and S.-H. Huang, "Mathematical modeling of cytotoxic lymphocyte-mediated immune response to hepatitis B virus infection," Journal of Biomedicine and Biotechnology, vol. 2008, Article ID 743690, 9 pages, 2008.

[4] G. Bocharov, B. Ludewig, A. Bertoletti et al., "Underwhelming the immune response: effect of slow virus growth on CD $8^{+}-\mathrm{T}-$ Lymphocytes responses," Journal of Virology, vol. 78, no. 5, pp. 2247-2254, 2004.

[5] A. M. Elaiw, R. M. Abukwaik, and E. O. Alzahrani, "Global properties of a cell mediated immunity in HIV infection model with two classes of target cells and distributed delays," International Journal of Biomathematics, vol. 7, no. 5, Article ID 1450055, 25 pages, 2014.

[6] A. M. Elaiw and A. M. Shehata, "Stability and feedback stabilization of HIV infection model with two classes of target cells," Discrete Dynamics in Nature and Society, vol. 2012, Article ID 963864, 20 pages, 2012.

[7] K. Hattaf and N. Yousfi, "Optimal control of a delayed HIV infection model with immune response using an efficient numerical method," ISRN Biomathematics, vol. 2012, Article ID 215124, 7 pages, 2012.

[8] K. Hattaf, N. Yousfi, and A. Tridane, "Global stability analysis of a generalized virus dynamics model with the immune response," Canadian Applied Mathematics Quarterly, vol. 20, no. 4, pp. 499-518, 2012.

[9] X. Shi, X. Zhou, and X. Song, "Dynamical behavior of a delay virus dynamics model with CTL immune response," Nonlinear Analysis: Real World Applications, vol. 11, no. 3, pp. 1795-1809, 2010.

[10] N. Yousfi, K. Hattaf, and A. Tridane, "Modeling the adaptive immune response in HBV infection," Journal of Mathematical Biology, vol. 63, no. 5, pp. 933-957, 2011.

[11] L. Min, Y. Su, and Y. Kuang, "Mathematical analysis of a basic virus infection model with application to HBV infection," Rocky Mountain Journal of Mathematics, vol. 38, no. 5, pp. 1573-1585, 2008.

[12] I. S. Gradshteyn and I. M. Ryzhik, "Routh-Hurwitz theorem," in Tables of Integrals, Series, and Products, Academic Press, San Diego, Calif, USA, 2000.

[13] J. P. LaSalle, The Stability of Dynamical Systems, Regional Conference Series in Applied Mathematics, SIAM, Philadelphia, Pa, USA, 1976.
[14] M. Y. Li and J. S. Muldowney, "A geometric approach to globalstability problems," SIAM Journal on Mathematical Analysis, vol. 27, no. 4, pp. 1070-1083, 1996.

[15] B. Buonomo and D. Lacitignola, "On the use of the geometric approach to global stability for three dimensional ODE systems: a bilinear case," Journal of Mathematical Analysis and Applications, vol. 348, no. 1, pp. 255-266, 2008.

[16] K. Wang, A. Fan, and A. Torres, "Global properties of an improved hepatitis B virus model," Nonlinear Analysis: Real World Applications, vol. 11, no. 4, pp. 3131-3138, 2010.

[17] K. Hattaf and N. Yousfi, "Global stability of a virus dynamics model with cure rate and absorption," Journal of the Egyptian Mathematical Society, 2014.

[18] V. Hutson and K. Schmitt, "Permanence and the dynamics of biological systems," Mathematical Biosciences, vol. 111, no. 1, pp. 1-71, 1992.

[19] H. I. Freedman, S. G. Ruan, and M. X. Tang, "Uniform persistence and flows near a closed positively invariant set," Journal of Dynamics and Differential Equations, vol. 6, no. 4, pp. 583-600, 1994.

[20] R. H. Martin Jr., "Logarithmic norms and projections applied to linear differential systems," Journal of Mathematical Analysis and Applications, vol. 45, pp. 432-454, 1974.

[21] R. M. Ribeiro, A. Lo, and A. S. Perelson, "Dynamics of hepatitis B virus infection," Microbes and Infection, vol. 4, no. 8, pp. 829$835,2002$. 


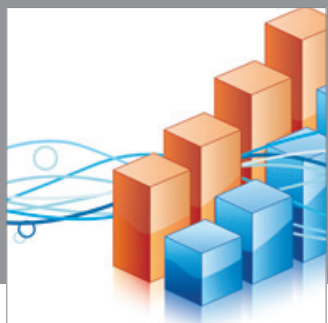

Advances in

Operations Research

mansans

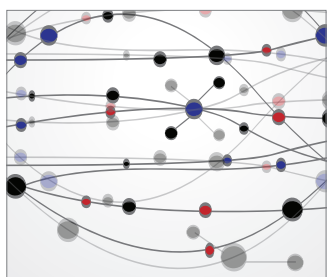

The Scientific World Journal
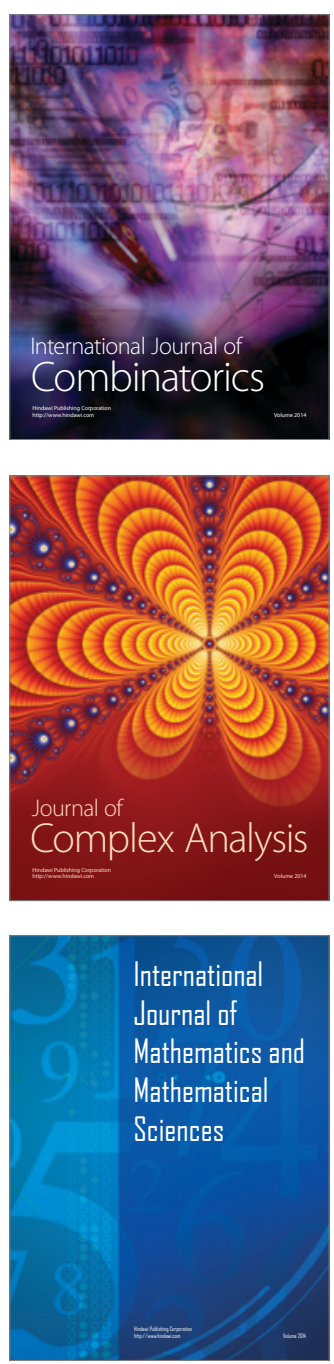
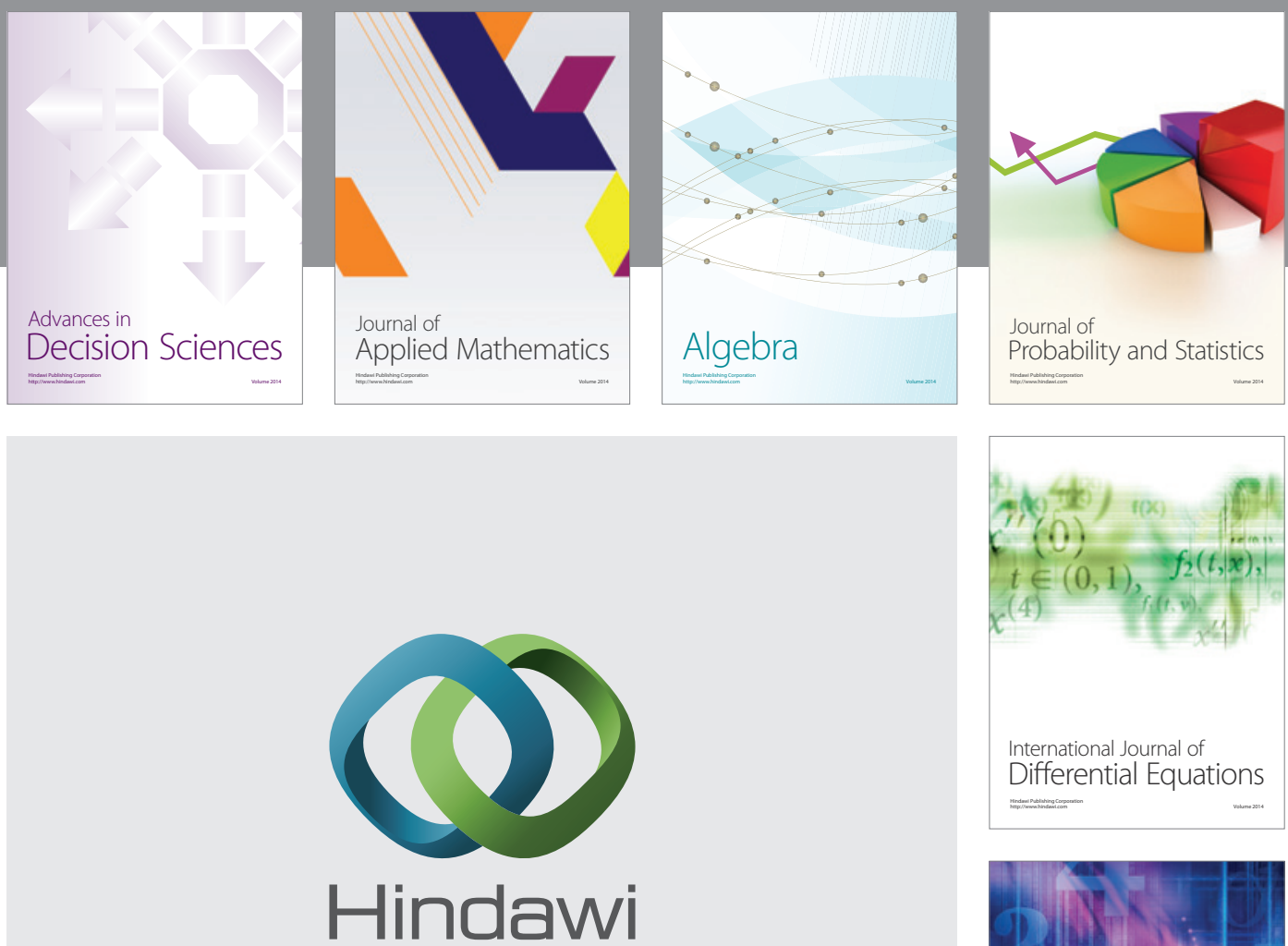

Submit your manuscripts at http://www.hindawi.com
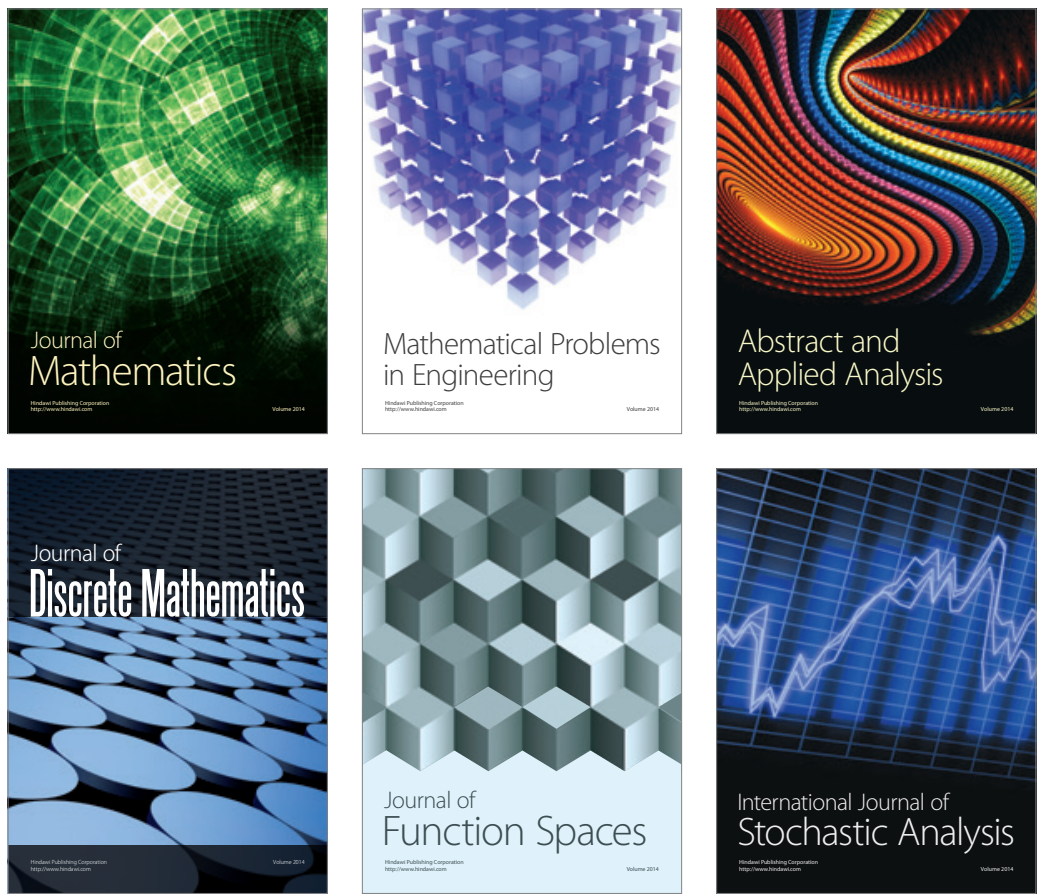

Journal of

Function Spaces

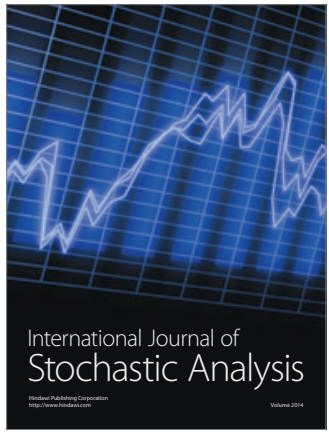

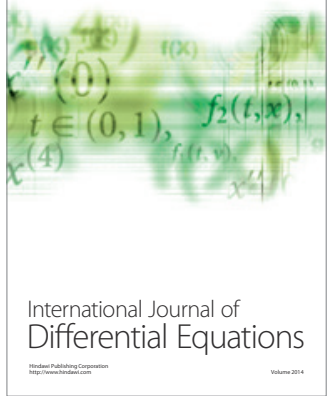
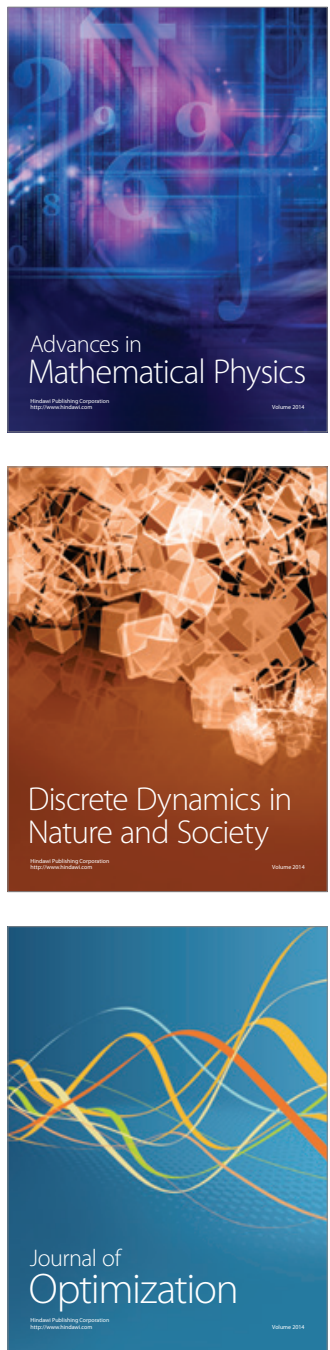